\title{
AN OPTIMIZATION APPROACH FOR THE EIGENVECTOR METHOD
}

\begin{abstract}
Pairwise comparison matrices play an important role in multiattribute decision making; they are applied to derive priorities or implicit weights for a given set of decision alternatives. Several approaches exist regarding how to derive a suitable vector of weights from a pairwise comparison matrix. Saaty proposed the eigenvector method in which the principal eigenvector of the pairwise comparison matrix serves as the vector of weights. Another class of approaches is based on optimization methods and proposes different ways for minimizing the difference between the pairwise comparison matrix and the consistent matrix constructed from the weights. In the talk, we show that the eigenvector method also can be considered from the aspect of the optimization approach. Namely, the principal eigenvector can be obtained from the optimal solution of a convex optimization problem. We show that this approach is very useful in the case of incomplete pairwise comparison matrices. Computational experience is also presented.
\end{abstract}

Keywords: pairwise comparison matrix, eigenvector method, convex optimization

The eigenvector method proposed by Saaty [3] is one of the widely used methods for eliciting the vector $w$ of the priority weights from an $n \times n$ pairwise comparison matrix $A$. The method determines the maximal eigenvalue $\lambda_{\max }$ of $A$, and the unique positive principal eigenvector fulfilling $A w=\lambda_{\max } w$ is considered as the priority vector derived from $A$.

Another approach is the class of the optimization methods. These methods aim at finding approximations $a_{i j} \approx w_{i} / w_{j}$. The basic idea is to construct a suitable function $\Phi$ of $A$ and $w$ somehow measuring the discrepancies between $a_{i j}$ and $w_{i} / w_{j}, i, j=1, \ldots, n$. Natural assumptions about this function $\Phi$ are as follows:

$\Phi(A ; w) \geq 0$

$\Phi(A ; w)=0$ if and only if $a_{i j}=w_{i} / w_{j}, i, j=1, \ldots, n$.

The optimization problem to be solved is then

$$
\min _{w>0} \Phi(A ; w) .
$$

Several methods of the optimization approach can be written in this form, e.g. the least squares method, the logarithmic least squares method, the weighted least squares method, the chi square method, just to mention some of them.

In the talk, we show that the eigenvector method also can be considered from the aspect of the optimization approach. We construct a function $\Phi$ such that by solving (1) we obtain the maximal eigenvalue and the principal eigenvector of $A$. It will be pointed out that (1) can be transformed into the form of a convex optimization problem. The idea can 
be extended to the problem of finding the principal eigenvector of the supermatrix in ANP [4], as well.

In [1], a method based on convex optimization was proposed for the optimal completion of incomplete pairwise comparison matrices. The method works as follows. Positive variables $x_{1}, \ldots, x_{d}$ are introduced for the missing elements in the upper triangular part of $\mathrm{A}$, and their reciprocals $1 / x_{1}, \ldots, 1 / x_{d}$ are written in the lower triangular part of $A$. Let $A(x)=A\left(x_{1}, \ldots, x_{d}\right)$ denote the matrix filled in by these variables. Let $x^{*}$ be an optimal solution of

$$
\min _{x>0} \lambda_{\max }(A(x))
$$

and let the missing positions be filled in with the corresponding components of $x^{*}$. This is a positive attitude, since the missing positions are filled in resulting in the lowest inconsistency index and ratio among the possible ones. Let

$$
y=\log x=\left(\log x_{1}, \ldots, \log x_{d}\right)^{T} .
$$

As shown in [1], $\lambda_{\max }(A(\exp y))$ is a logconvex (hence convex) function of $y$. Consequently, problem (2) is equivalent to the unconstrained convex minimization problem

$$
\min \lambda_{\max }(A(\exp y)) .
$$

The optimal solution of (3) is unique if and only if the matrix with zeros in the missing positions is irreducible.

When applying convex optimization techniques for solving (3), it may cause a numerical difficulty that $\lambda_{\max }(A(\exp y))$ can be evaluated only approximately by iterative way at any search point.

In the second part of the talk we show that the convex optimization approach proposed for computing $\lambda_{\max }(A)$ can be integrated into (3) resulting in a single convex optimization problem. The main advantage is that $\lambda_{\max }$ does not need to be evaluated during the solution process. In addition, sophisticated nonlinear optimization packages can be used even for large problems

Finally, computational experience will be shown on large incomplete pairwise comparison matrices presented in [2]. In these optimization problems, we had $n=149$ and $d=10,216$. The problems were solved by using GAMS/MINOS.

\section{References}

1. Bozóki, S., Fülöp, J., \& Rónyai, L. (2010). On optimal completion of incomplete pairwise comparison matrices. Mathematical and Computer Modelling, 52(1-2), $318-333$.

2. Csató, L. (2013). Ranking by pairwise comparisons for Swiss-system tournaments. Central European Journal of Operations Research, 21(4), 783-803.

3. Saaty, T.L. (1980). The analytic hierarchy process. McGraw-Hill, New York.

4. Saaty, T.L. (2001). Decision Making with Dependence and Feedback: The Analytic Network Process (second ed.). RWS Pub., Pittsburg. 